\title{
Inhibitory effects of Ponciri Fructus on testosterone-induced benign prostatic hyperplasia in rats
}

\author{
Woo-Young Jeon ${ }^{1} \mathbb{B}$, Ohn Soon Kim², Chang-Seob Seo ${ }^{1}$, Seong Eun Jin ${ }^{1}$, Jung-Ae Kim³, Hyeun-Kyoo Shin',
} Yong-ung $\mathrm{Kim}^{4^{*}}$ and Mee-Young Lee ${ }^{1 *}$

\begin{abstract}
Background: Benign prostatic hyperplasia (BPH) is non-cancerous condition of enlargement of the prostate, a common occurrence in older men. The immature fruits of Poncirus trifoliata (L.) Rafinesque (Rutaceae), Ponciri Fructus are widely used in traditional oriental medicine for the therapy of various diseases. However, little is known about the mechanism underlying the pathogenesis of $\mathrm{BPH}$. In the present study, we investigated the protective effects of a Ponciri Fructus extract (PFE) on the development of BPH in a in a rat model of BPH induced by testosterone propionate (TP).
\end{abstract}

Methods: Male Sprague Dawley rats were used as a model of BPH after its induction by daily subcutaneous injections of TP/corn oil, for a period of four weeks. PFE was administrated daily $1 \mathrm{~h}$ before TP/corn oil injection by oral gavage at a dose level of $200 \mathrm{mg} / \mathrm{kg}$ during the 4 weeks of TP/corn oil injections. All rats were sacrificed at the end of the experiment, we measured the relative prostate weight, the levels of testosterone and dihydrotestosterone (DHT), histological changes, activities of antioxidant enzymes (catalase, glutathione peroxidase, glutathione reductase, and superoxide dismutase), and expression of proliferating cell nuclear antigen (PCNA). In addition, we also measured the inhibition (\%) of 5a-reductase in the prostatic tissue.

Results: Our findings indicate that PFE significantly inhibited the development of $\mathrm{BPH}$; decreased the relative prostate weight, the level of testosterone and DHT in serum and prostatic tissue, prostatic hyperplasia, expression of PCNA, and increased the antioxidant enzymes. Moreover, PFE showed a weak inhibitory activity on 5a-reductase.

Conclusions: These results suggest that PFE may be used as a therapeutic agent for BPH via antiproliferative and antioxidant effects.

Keywords: Ponciri Fructus, Benign prostatic hyperplasia, Dihydrotestosterone, Antioxidant enzymes, Proliferating cell nuclear antigen

\section{Background}

Benign prostatic hyperplasia (BPH) is one of the most common diseases of aging in men and is characterized by progressive hyperplasia of glandular and stromal tissues, leading to an enlarged prostate size [1]. An enlarged prostate can constrict the urethra, leading to severe urinary problems including a weak urinary stream, dysuria,

\footnotetext{
* Correspondence: ykim@dhu.ac.kr; cozy11@kiom.re.kr

${ }^{4}$ Department of Pharmaceutical Engineering, College of Biomedical Science

Daegu Haany University, 1 Hanuidae-ro, Gyeongsan-si, Gyeongsangbuk-do 38610, Republic of Korea

'K-herb Research Center, Korea Institute of Oriental Medicine, 1672

Yuseong-daero, Yuseong-gu, Daejeon 34054, Republic of Korea

Full list of author information is available at the end of the article
}

urinary frequency, bladder outlet obstruction, and incomplete bladder emptying [2]. Biochemically, an imbalance of androgen/estrogen and overexpression of growth factors plays an important part in the development and progression of BPH [3]. Dihydrotestosterone (DHT), a metabolite of testosterone and a crucial mediator of prostate growth, is synthesized in the prostate from circulating testosterone by $5 \alpha$-reductase and is a highly lipophilic enzyme found on intracellular membranes [4]. $5 \alpha$-reductase exists in two isoforms: Type 1 and Type 2. Finasteride, a synthetic $5 \alpha-$ reductase Type 2 inhibitor, is used to treat $\mathrm{BPH}$ [5].

Oxidative stress plays a critical role in prostatic hyperplasia and is responsible for an imbalance between the 
production of free radicals and scavenging of free radicals, and can injure important components of tissues such as mRNA, DNA, and proteins [6]. Many studies have investigated antioxidants in the prevention and treatment of $\mathrm{BPH}$ $[7,8]$, and antioxidants have been proposed as therapeutic agents to prevent the progress of $\mathrm{BPH}$ by increasing the activities of antioxidant enzymes such as catalase (CAT), glutathione peroxidase (GPx), glutathione reductase (GR), and superoxide dismutase (SOD).

The immature fruits of Poncirus trifoliata (L.) Rafinesque (Rutaceae), Ponciri Fructus are traditionally used for the treatment of common edema, dyspepsia, and constipation in many Asian countries [9]. Many pharmacological studies have shown that Ponciri Fructus exerts anti-inflammatory [10] and gastroprotective effects [11] in vitro and in vivo. Ponciri Fructus contains high amounts of various compounds, including flavonoids and coumarins [11]. They demonstrate an extensive range of biological activities including antiallergic, antitumor, antiviral, and cancer chemopreventive properties. In the present study, in vivo experiments were performed to confirm the effect of Ponciri Fructus extract (PFE) in an animal model of BPH, because its effect has proved in vitro using a myofibroblast stromal cell line-derived from normal adult prostate (WPMY-1) in our laboratory (data not shown). Although many studies have investigated the pharmacological effects of PFE, there is little information regarding its effects on the mechanism underlying the pathogenesis of $\mathrm{BPH}$. Therefore, we aimed to investigate the antiproliferative and antioxidant effects of PFE related to oxidative stress on the development of $\mathrm{BPH}$ in a rat model of $\mathrm{BPH}$ induced by testosterone propionate (TP).

\section{Methods}

\section{Plant materials}

Dried fruit of $P$. trifoliata were purchased from Kwangmyungdang (Ulsan, Korea) in November 2014 and taxonomically confirmed by Dr. Jung Hoon Kim at the Division of Pharmacology, School of Korean Medicine, Pusan National University (Yangsan, Gyeongnam 626870, Korea). A voucher specimen (2014-GO-10) has been deposited at the K-herb Research Center, Korea Institute of Oriental Medicine (KIOM).

\section{Chemicals and reagents}

Neoponcirin, poncirin, and auraptene were purchased from ChemFaces (Wuhan, China). Umbelliferone, naringin, and imperatorin were obtained from Shanghai Sunny Biotech (Shanghai China), Sigma-Aldrich (St Louis, MO, USA), and ChromaDex (Irvine, CA, USA), respectively. The purity of the six reference standards was $\geq 95.0 \%$. HPLC-grade methanol, acetonitrile, and water were obtained from J.T. Baker (Phillipsburg, NJ, USA). Glacial acetic acid, analytical reagent grade was purchased from
Merck (Darmstadt, Germany). Other reagents were as follows: TP (T0028; Tokyo Chemical Ins. Co., Tokyo, Japan), corn oil (C8267; Sigma-Aldrich), (-)-riboflavin (Rib) (R9504; Sigma-Aldrich), Finasteride (Fin) (F1293; SigmaAldrich), testosterone enzyme-linked immunosorbent assay (ELISA) kit (582,701; Cayman Chemical, Ann Arbor, MI, USA), DHT ELISA kit (11-DHTHU-E01; ALPCO Diagnostics, Salem, NH, USA), catalase assay kit (707,002; Cayman Chemical), GPx assay kit (703,102; Cayman Chemical), GR assay kit (703,202; Cayman Chemical), SOD assay kit (706,002, Cayman Chemical), anti-proliferating cell nuclear antigen (PCNA) antibody (ab-29; Abcam, Cambridge, MA, USA) and anti- $\beta$-actin antibody (4967S; Cell Signaling Technology, Danvers, MA, USA).

\section{Preparation of $70 \%$ ethanol extract of Ponciri fructus}

Dried fruit of $P$. trifoliata $(100.0 \mathrm{~kg})$ were extracted with $70 \%$ ethanol (1000 L $\times 3$ times) for $60 \mathrm{~min}$ at $80{ }^{\circ} \mathrm{C}$ using and electric extractor (Cosmos-660; Kyungseo Machine Co., Incheon, Korea). The extracted solution was filtered through filter paper, evaporated to dryness at $40{ }^{\circ} \mathrm{C}$ under vacuum (Eyela N-21NS, Tokyo, Japan), and freeze-dried (PVTFD10RS, IlShinBioBase, Yangju, Korea). The amount of $70 \%$ ethanolic extract obtained was $19.4 \mathrm{~kg}(19.4 \%)$.

\section{High-performance liquid chromatography (HPLC) analysis}

The chromatographic analysis for simultaneous quantification of the three flavonoids (naringin, neoponcirin, and poncirin) and the three coumarins (umbelliferone, imperatorin, and auraptene) was performed using a Prominence LC-20A series (Shimadzu Co., Kyoto, Japan) consisting of a solvent delivery unit (LC-20AT), online degasser (DGU$20 \mathrm{~A}_{3}$ ), column oven (CTO-20A), auto sample injector (SIL-20 AC), and photodiode array detector (PDA, SPDM20A) as described previously [12]. The data acquired were processed using Lab Solution software (version 5.54 SP3, Shimadzu, Kyoto, Japan). Six compounds were separated on a SunFire C18 column $(250 \mathrm{~mm} \times 4.6 \mathrm{~mm}$, $5 \mu \mathrm{m}$, Waters, Milford, MA, USA) maintained at $40{ }^{\circ} \mathrm{C}$. The mobile phases consisted of $1.0 \%(v / \mathrm{v})$ acetic acid in distilled water $(\mathrm{A})$ and $1.0 \%(\mathrm{v} / \mathrm{v})$ acetic acid in acetonitrile (B). The gradient flow was as follows: $10 \%-65 \%$ B for $0-$ $30 \mathrm{~min}, 65 \%-90 \%$ B for $30-35 \mathrm{~min}, 90 \%$ B for $35-40 \mathrm{~min}$, and $90 \%-10 \%$ B for $40-45 \mathrm{~min}$. The flow-rate was $1.0 \mathrm{~mL} /$ min and the injection volume was $10 \mu \mathrm{L}$. For quantitative determination, $100 \mathrm{mg}$ of lyophilized sample was dissolved in $20 \mathrm{~mL}$ of distilled $70 \%$ methanol and then the solution was filtered through a $0.2 \mu \mathrm{m}$ membrane filter (PALL Life Sciences, Ann Arbor, MI, USA) before HPLC.

\section{Animals}

Male 6-week-old Sprague Dawley (SD) rats weighing 250-350 g (Orientbio Inc., Seoul, Korea) were housed in a room maintained at $18-23{ }^{\circ} \mathrm{C}$ with a relative humidity 
of $40-60 \%$, and an alternating $12 \mathrm{~h} \mathrm{light} / 12 \mathrm{~h}$ dark cycle. Rats were provided with a standard laboratory diet and water ad libitum. All experimental procedures were conducted in accordance with the NIH Guidelines for the Care and Use of Laboratory Animals and were approved by the Institutional Animal Care and Use Committee of the Chungnam National University (animal ethics approval number: CNU-00446). Animal handling followed the dictates of the National Animal Welfare Law of Korea.

\section{Experimental procedures}

The design of this experiment is outlined in Fig. 1. BPH was induced by subcutaneous injection of TP $(3 \mathrm{mg} / \mathrm{kg})$ for 4 weeks. After 1 week of acclimatization, the rats were randomly divided into four groups ( $n=6$ per group): (A) Normal control group (NC group), corn oil injection (subcutaneously, s.c.) + phosphate buffered saline (PBS) administration (peroral, p.o.); (B) control group (BPH group), TP (3 $\mathrm{mg} / \mathrm{kg}$ )/corn oil injection (s.c.) + PBS administration (p.o.); (C) positive control group (Fin-treated group), TP (3 $\mathrm{mg} / \mathrm{kg}) /$ corn oil injection (s.c.) + finasteride administration (10 mg/kg, p.o.); (D) experimental group (PFE-treated group), TP (3 mg/kg)/corn oil injection (s.c.) + PFE administration (200 mg/kg, p.o.). Finasteride, a $5 \alpha-$ reductase inhibitor and anti-BPH drug, was used as a positive control [13].

All materials were administered to rats once daily for 4 weeks, and body weight was measured weekly. The application volumes were $5 \mathrm{~mL} / \mathrm{kg}$ for oral administration (PBS, finasteride and PFE) and $1 \mathrm{~mL} / \mathrm{kg}$ for subcutaneous injection (corn oil and TP/corn oil) and were calculated in advance based on the most recently recorded body weights of individual rats. After the final treatment, all rats were fasted overnight and anesthetized using pentobarbital $100 \mathrm{mg} / \mathrm{kg}$ body weight injected intraperitoneally (Han Lim Pharmaceutical. Co. Ltd., Yongin, Korea). Blood samples were drawn from the caudal vena cava, and the

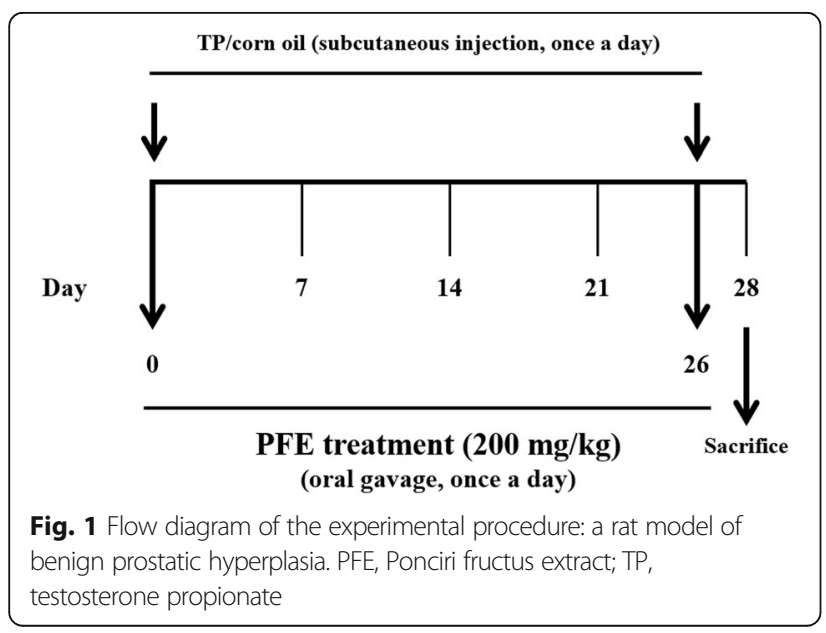

serum was separated by centrifugation (1000 g for $15 \mathrm{~min}$, $\left.4{ }^{\circ} \mathrm{C}\right)$. Serum was stored at $-80{ }^{\circ} \mathrm{C}$ for hormone assays. The prostates were removed immediately and weighed. Relative prostate weight was calculated as the ratio of prostate weight to body weight. The percentage inhibition of the increase in prostate weight induced by PFE was determined in accordance with a previously described method [14]. The ventral lobe of the prostate was divided in half. One half was fixed using $4 \%$ paraformaldehyde (sc-281,692; Santa Cruz Biotechnology, Santa Cruz, CA, USA) and embedded in paraffin for histomorphology and the other was stored at $-80^{\circ} \mathrm{C}$ for other analyses.

\section{Preparation of prostate homogenates}

Prostatic tissue was homogenized $(1 / 10 \mathrm{w} / v)$ in tissue lysis/extraction reagent (C3228; Sigma-Aldrich) containing protease inhibitor cocktail (Ref 11836153 001; Roche, Mannheim, Germany) using a homogenizer (T10 Basic Ultra Turrax; IKA Works, Staufen, Germany). Homogenates were centrifuged at $15,500 \mathrm{~g}$ for $20 \mathrm{~min}$ at $4{ }^{\circ} \mathrm{C}$. Total protein concentrations in the supernatant fractions were measured using Bradford reagent (No. 500-0006; Bio-Rad Laboratories, Inc., Hercules, CA, USA) as described previously [13].

\section{Elisa}

The levels of testosterone and DHT in the serum or prostatic tissue were determined using an ELISA kit according to the manufacturer's instructions. The absorbance was measured at $450 \mathrm{~nm}$ using a microplate ELISA reader (Bio-Rad Laboratories, Inc.). Values are expressed per $\mathrm{mL}$ for serum and per mg protein for the prostatic tissue.

\section{Histological examination}

Fixed prostatic tissue embedded in paraffin wax was cut into $4 \mu \mathrm{m}$ thick sections and stained with hematoxylin (MHS-16; Sigma-Aldrich) and eosin (HT110-1-32; SigmaAldrich). Coverslips were mounted on sections using mounting medium (Invitrogen, Carlsbad, CA, USA) and then the sections were examined under a microscope (Nikon, Tokyo, Japan). Prostatic epithelial thickness was measured using an image analyzer (Molecular Devices Inc., CA, USA) as described previously [13].

\section{Antioxidant assay}

The activities of antioxidant enzymes, including CAT, GPx, GR, and SOD were quantified using commercial kits according to the manufacturer's protocols and the results were expressed as $\mathrm{U} / \mathrm{mg}$ protein as described previously [15].

\section{Immunoblotting}

Equal amounts of total prostatic protein $(30 \mu \mathrm{g})$ were heated at $100{ }^{\circ} \mathrm{C}$ for $5 \mathrm{~min}$, loaded onto $8 \%$ sodium 
dodecyl sulfate-polyacrylamide gels, and electrophoresed (at $100 \mathrm{~V}$ for $90 \mathrm{~min}$ ). The proteins were then transferred to an Immobilon-P polyvinylidene difluoride membrane (IPVH00010; Millipore Corporation, Bedford, MA, USA) at $25 \mathrm{~V}$ for $30 \mathrm{~min}$ using Trans-Blot Turbo Transfer System (Bio-Rad Laboratories). The membrane was blocked for 60 min with Tris-buffered saline containing 0.05\% Tween-20 (TBST) plus 5\% skim milk (REF 232100; BD Difco, Sparks, MD, USA), followed by incubation with anti-PCNA (1:1000 dilution), and anti- $\beta$-actin (1:1000 dilution) antibodies overnight at $4{ }^{\circ} \mathrm{C}$. The membrane was washed three times with TBST at intervals of $10 \mathrm{~min}$ and then incubated with a horseradish peroxidase (HRP)-conjugated secondary antibody (PCNA, anti-mouse; and $\beta$ actin, anti-rabbit; 1:3000 dilution, respectively) (Jackson ImmunoResearch, West Grove, PA, USA) for $60 \mathrm{~min}$ at room temperature. The membrane was washed three times with TBST at intervals of $10 \mathrm{~min}$ and developed using the SuperSignal West Femto Maximum Sensitivity Substrate (ultrasensitive enhanced chemiluminescent substrate) (NCI34095KR; Thermo Fisher Scientific Inc., Waltham, MA, USA). Subsequently, membranes were photographed and, densitometric band values were determined using the commercially available ChemiDoc $\mathrm{XRS}^{+}$Imaging System for quantitative analyses (Bio-Rad Laboratories) as described previously [16].

\section{5a-reductase activity}

In an additional experiment, a suspension of testosterone $5 \alpha$-reductase was prepared from the homogenate of the ventral prostates of male SD rats, and the inhibition (\%) of $5 \alpha$-reductase was measured according to a method previously reported [17]. Both the Type 1 and Type $25 \alpha-$ reductase isozymes are present in the ventral prostate of rats [18]. Since the extracts of rat prostate for measurement the inhibition of $5 \alpha$-reductase were used in a neutral $\mathrm{pH}$ buffer, $5 \alpha$-reductase inhibition (\%) was applied to inhibitory experiments using $5 \alpha$-reductase inhibitors. Each enzyme reaction was conducted in duplicate.

\section{Statistical analysis}

All data are presented as the mean \pm standard error of the mean (SEM). Statistical significance was determined using analysis of variance (ANOVA) followed by a multiple comparison procedure and a Dunnett post hoc test. Differences in $P$ values $<0.05$ or $<0.01$ were considered statistically significant [19].

\section{Results}

HPLC analysis of PFE for three flavonoids and three coumarins

An established HPLC-PDA analytical method was used for the simultaneous analysis of three flavonoids (naringin, neoponcirin, and poncirin) and three coumarins (umbelliferone, imperatorin, and auraptene) in the PFE. All components were separated within 45 min using an optimized analytical method and representative three-dimensional chromatograms are shown in Fig. 2. The retention times of umbelliferone, naringin, neoponcirin, poncirin, imperatorin, and auraptene were 14.22, 14.77, 18.86, 19.37, 33.58, and $40.66 \mathrm{~min}$, respectively. The correlation coefficients with the six authentic standards in the tested concentration ranges measured were $\geq 0.9997$. Under these optimized analytical conditions, the contents of three flavonoids (naringin, neoponcirin, and poncirin) and the three coumarins (umbelliferone, imperatorin, and auraptene) in the lyophilized sample were detected at $1.17,89.20,28.94,211.36,2.25$, and $15.92 \mathrm{mg} / \mathrm{g}$, respectively.

\section{Effect of PFE on the prostate weight}

Relative prostate weight is commonly used to evaluate the development of BPH. Rats in the TP-induced BPH group $(0.0046 \pm 0.0007, P<0.01)$ showed relative prostate weights that were significantly greater than those of rats in the NC group $(0.0019 \pm 0.0002)$, whereas prostate weights of rats in the Fin-treated group $(0.0032 \pm 0.0004, P<0.01)$ were decreased markedly compared with those in the $\mathrm{BPH}$ group. The PFE-treated group $(0.0039 \pm 0.0004, P<0.05)$ also showed significant decreases in relative prostate weights compared with the $\mathrm{BPH}$ group. These results were similar to those for the Fin-treated group (Fig. 3).

\section{Effect of PFE on the levels of testosterone and DHT in serum and prostatic tissue}

The major prostatic androgen is DHT, which is formed by the SRD5A2 from the influence of testosterone. As shown in Fig. 4a, rats in the TP-induced $\mathrm{BPH}$ group $(1.36 \pm 0.07 \mathrm{ng} / \mathrm{mL}, P<0.01)$ showed a significant increase in serum testosterone level compared with the $\mathrm{NC}$ group $(0.42 \pm 0.17 \mathrm{ng} / \mathrm{mL})$. By contrast, rats in the Fin-treated group $(0.98 \pm 0.14 \mathrm{ng} / \mathrm{mL}, P<0.01)$ showed a significantly reduced serum testosterone level compared with those in the $\mathrm{BPH}$ group. Like rats in the Fin-treated group, those in the PFE-treated group $(1.09 \pm 0.24 \mathrm{ng} / \mathrm{mL}$, not significant) showed a marked reduction in testosterone level compared with the BPH group.

The DHT level $(254.56 \pm 39.50 \mathrm{pg} / \mathrm{mg}$ protein, $P<0.01)$ in the prostates of rats in the TP-induced $\mathrm{BPH}$ group was markedly higher than in rats in the $\mathrm{NC}$ group $(149.09 \pm 12.60 \mathrm{pg} / \mathrm{mg}$ protein). However, prostatic DHT level in rats in the Fin-treated group $(180.38 \pm 14.81 \mathrm{pg} / \mathrm{mg}$ protein, $P<0.01$ ) was significantly lower than in the $\mathrm{BPH}$ group. The levels of prostatic DHT in rats in the PFEtreated group $(197.17 \pm 43.05 \mathrm{pg} / \mathrm{mg}$ protein, $P<0.05)$ were significantly less than the levels in rats from the $\mathrm{BPH}$ group. These results were similar to those for the Fintreated group (Fig. 4b). 


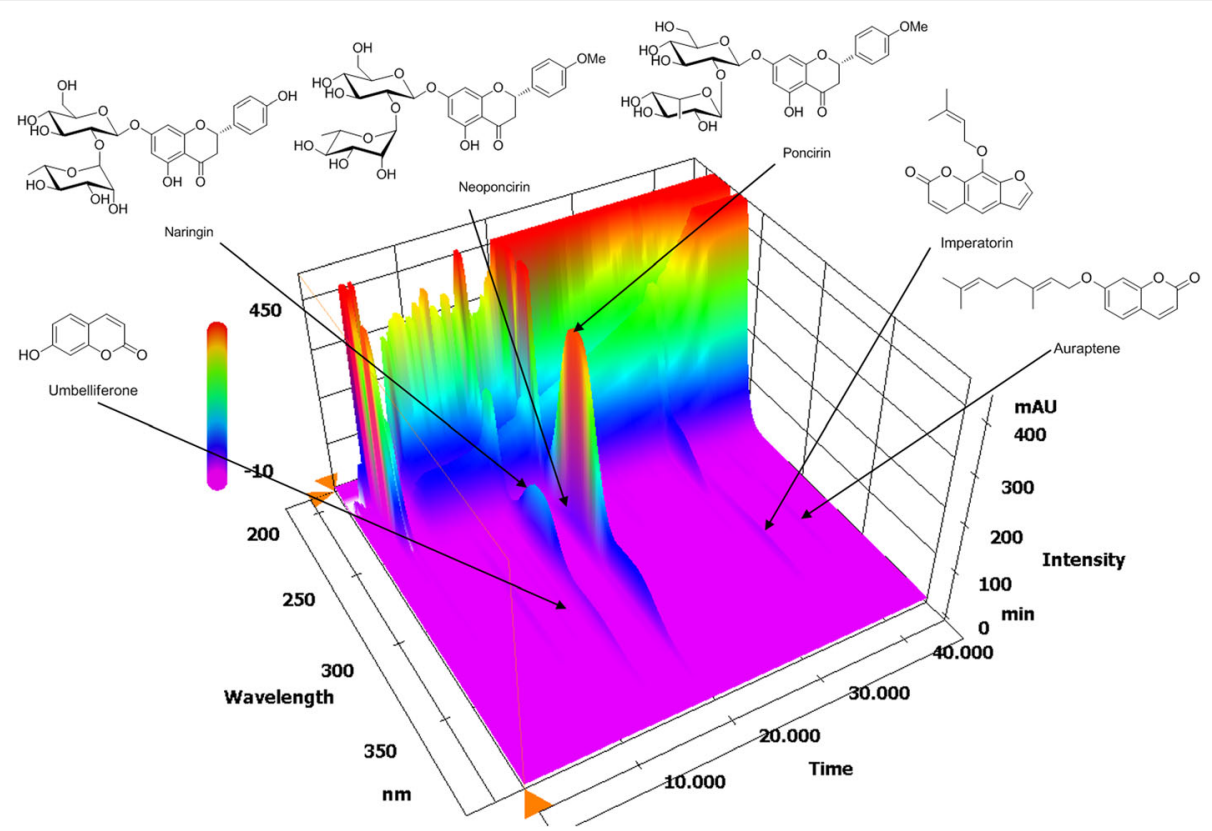

Fig. 2 Three-dimensional chromatogram of Ponciri fructus extract by HPLC-PDA. HPLC-PDA, high-performance liquid chromatography-photodiode array detector

\section{Effects of PFE on prostatic epithelial hyperplasia}

The development of epithelial hyperplasia was evaluated by histology of prostatic lesions. As show in Fig. 5, the epithelial cell layer and lumen spaces of the prostate were larger in rats from the TP-induced BPH group compared with those from the NC group. Rats from the Fin-treated

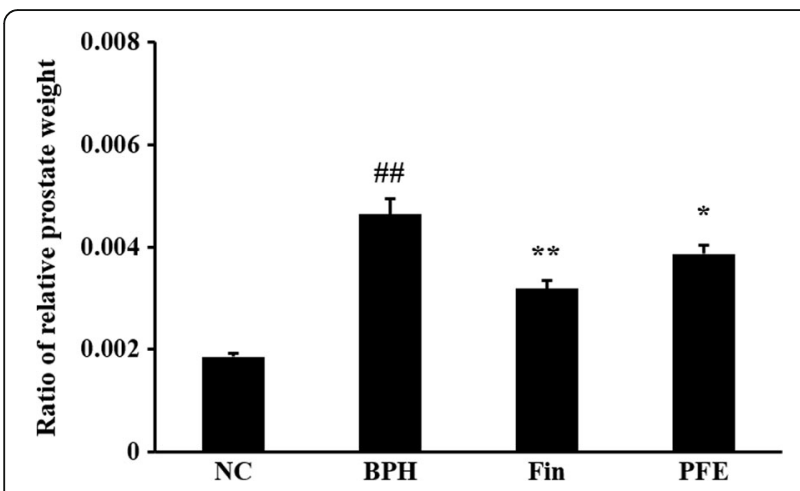

Fig. 3 Effect of PFE on prostate weight in rats with TP-induced BPH. Ratio of relative prostate weight is the ratio of prostate weight to body weight $(\mathrm{g} / \mathrm{g})$. NC (normal control group), corn oil injection (s.c.) + PBS administration (p.o.); BPH (control group), TP (3 mg/ $/ \mathrm{kg}) / \mathrm{corn}$ oil injection (s.c.) + PBS administration (p.o.); Fin (positive control group), TP (3 mg/kg)/corn oil injection (s.c.) + finasteride administration (10 mg/kg, p.o.); PFE (PFE-treated group), TP (3 mg/kg)/corn oil injection (s.c.) + PFE administration (200 $\mathrm{mg} / \mathrm{kg}$, p.o.). Data are presented as mean \pm S.E.M. $(n=6)$. Significant differences at ${ }^{\# \# P} P 0.01$ compared with the NC group. Significant differences at ${ }^{*} P<0.05$ and ${ }^{* *} P<0.01$ compared with the $\mathrm{BPH}$ group. $\mathrm{BPH}$, benign prostatic hyperplasia; Fin, finasteride; PBS, phosphate-buffered saline; PFE, Ponciri fructus extract; p.o., peroral; s.C., subcutaneously; TP, testosterone propionate group exhibited mild epithelial hyperplasia compared with those from the BPH group. Rats from the PFE-treated group also showed a reduction in epithelial hyperplasia compared with the BPH group. Rats from the BPH group exhibited greatly increased prostatic epithelial thickness compared with those from the NC group; however, rats from the PFE-treated and the Fin-treated groups exhibited markedly reduced hyperplasia compared with those from the $\mathrm{BPH}$ group.

\section{Effect of PFE on the activities of antioxidant enzymes in prostatic tissue}

To investigate the effects of PFE on the radical-scavenging antioxidant system in the TP-induced model of $\mathrm{BPH}$, antioxidant assays were performed. The activities of antioxidant enzymes including CAT, GPx, GR, and SOD were significantly lower in prostates from rats in the TP-induced BPH group $(20.09 \pm 3.72,44.30 \pm 17.41,9.64 \pm 2.29$, and $8.38 \pm 1.26 \mathrm{U} / \mathrm{mg}$ protein, respectively) compared with those from rats in the $\mathrm{NC}$ group (28.73 \pm 3.98 , $79.23 \pm 7.60,14.41 \pm 1.78$, and $12.71 \pm 1.53 \mathrm{U} / \mathrm{mg}$ protein, respectively). GPx and GR activities were significantly higher in prostates from rats the Fin-treated group $(73.71 \pm 11.37$ and $16.14 \pm 2.19 \mathrm{U} / \mathrm{mg}$ protein, respectively) compared with those from rats in the BPH group. CAT, GR, and SOD activities were significantly higher in rats in the PFE-treated group $(29.64 \pm 4.43,16.00 \pm 2.04$, and $11.82 \pm 1.70 \mathrm{U} / \mathrm{mg}$ protein, respectively) compared with rats in the BPH group. GPx activity was not significantly increased in prostates from rats in the PFE-treated group 

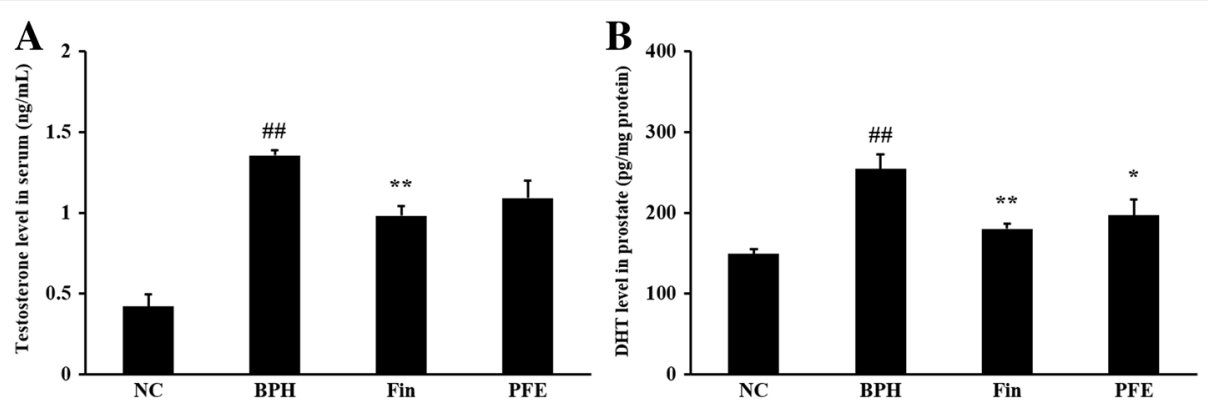

Fig. 4 Effect of PFE on the levels of testosterone $(\mathbf{a})$ and DHT (b) in serum and prostatic tissue. Individual data were obtained using an ELISA. NC (normal control group), corn oil injection (s.c.) + PBS administration (p.o); BPH (control group), TP (3 mg/kg)/corn oil injection (s.c.) + PBS administration (p.o.); Fin (positive control group), TP (3 mg/kg)/corn oil injection (s.c.) + finasteride administration (10 mg/kg, p.o.); PFE (PFE-treated group), TP (3 mg/kg)/ corn oil injection (s.C.) + PFE administration (200 mg/kg, p.o.). Data are presented as mean \pm S.E.M. $(n=6)$. Significant differences at ${ }^{\# \#} P<0.01$ compared with the NC group. Significant differences at ${ }^{*} P<0.05$ and ${ }^{* *} P<0.01$ compared with the BPH group. BPH, benign prostatic hyperplasia; DHT, dihydrotestosterone; Fin, finasteride; PBS, phosphate-buffered saline; PFE, Ponciri fructus extract; p.o., peroral; s.c., subcutaneously; TP, testosterone propionate

(58.88 $\pm 13.73 \mathrm{U} / \mathrm{mg}$ protein) compared with those from rats in the BPH group (Fig. $6 \mathrm{a}-\mathrm{d}$ ).

\section{Effect of PFE on the expression of PCNA in prostatic tissue}

To determine the correlation between the activation of PCNA and prostatic hyperplasia in the TP-induced model of $\mathrm{BPH}$, western blots were performed. As shown in Fig. 7a, the expression of PCNA protein was increased in prostates from rats in the TP-induced $\mathrm{BPH}$ group compared with those from rats in the NC group. Prostates from rats in the Fin-treated group had markedly reduced expression of PCNA compared with those from rats in the BPH group. Prostates from rats in the PFE-treated group also exhibited
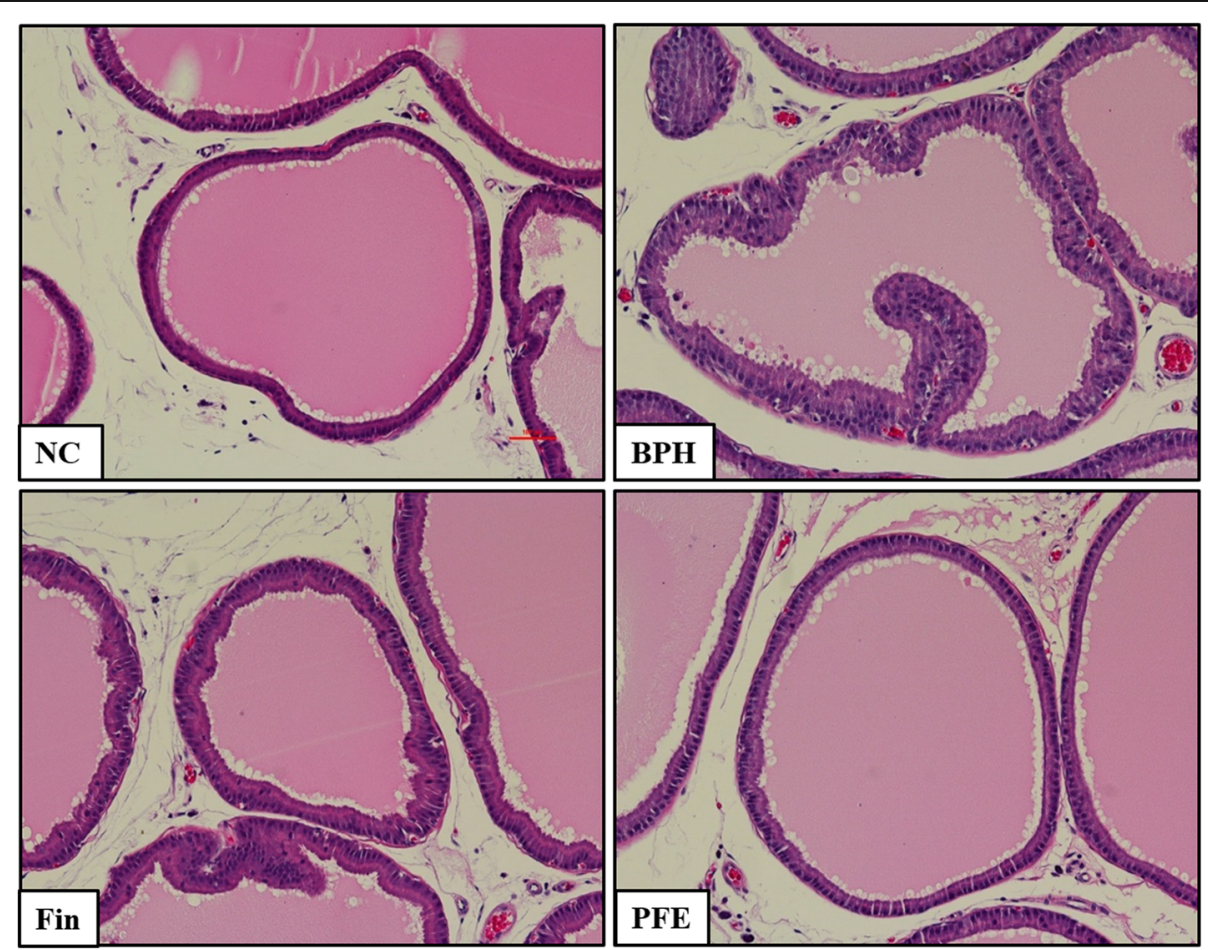

Fig. 5 Effect of PFE on the prostatic hyperplasia in TP-induced rats. Prostatic tissues were stained with a H\&E solution for histological examination (magnification, 200X). Representative photomicrographs of prostate sections are shown. NC (normal control group), corn oil injection (s.c.) + PBS administration (p.o.); BPH (control group), TP (3 mg/kg)/corn oil injection (s.c.) + PBS administration (p.o.); Fin (positive control group), TP (3 mg/kg)/ corn oil injection (s.c.) + finasteride administration (10 mg/ $/ \mathrm{kg}$, p.o.); PFE (PFE-treated group), TP (3 mg/kg)/corn oil injection (s.c.) + PFE administration (200 mg/kg, p.o.). BPH, benign prostatic hyperplasia; Fin, finasteride; H\&E, hematoxylin and eosin; PBS, phosphate-buffered saline; PFE, Ponciri fructus extract; p.o., peroral; s.c., subcutaneously; TP, testosterone propionate 

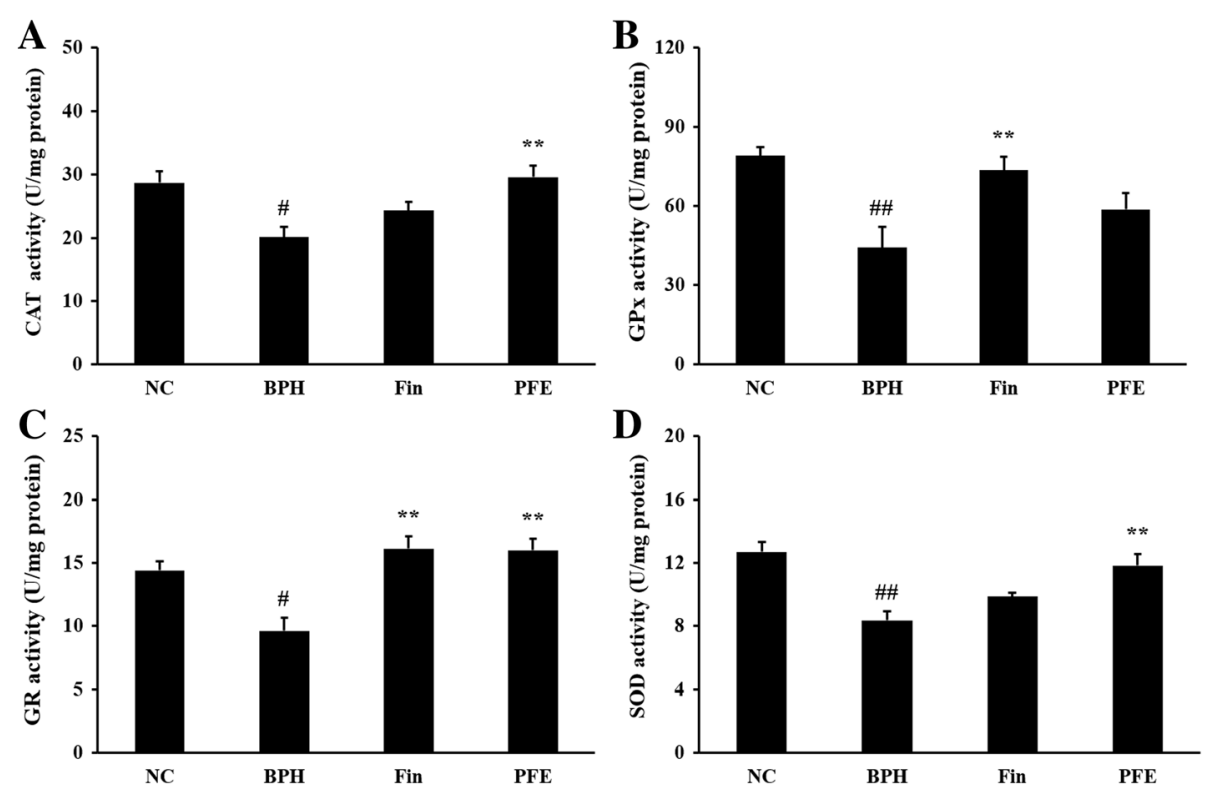

Fig. 6 Effect of PFE on the activities of antioxidant enzymes in prostatic tissue. Individual data were obtained using assay kits: (a) CAT, (b) GPX, (c) GR, and (d) SOD. NC (normal control group), corn oil injection (s.c.) + PBS administration (p.o.); BPH (control group), TP (3 mg/kg)/corn oil injection (s.c.) + PBS administration (p.o.); Fin (positive control group), TP (3 mg/kg)/corn oil injection (s.c.) + finasteride administration (10 mg/kg, p.o.); PFE (PFE-treated group), TP (3 mg/kg)/corn oil injection (s.c.) + PFE administration (200 mg/kg, p.o.). Data are presented as mean \pm S.E.M. $(n=6)$. Significant differences at ${ }^{\#} P<0.05$ and ${ }^{\# \#} P<0.01$ compared with the NC group. Significant differences at ${ }^{* *} P<0.01$ compared with the BPH group. BPH, benign prostatic hyperplasia; CAT, catalase; Fin, finasteride; GPx, glutathione peroxidase; GR, glutathione reductase; PBS, phosphatebuffered saline; PFE, Ponciri fructus extract; p.o., peroral; s.c., subcutaneously; SOD, superoxide dismutase; TP, testosterone propionate

a reduction in the expression of PCNA compared with those from rats in the BPH group, similar to what was observed in prostates from rats in the Fin-treated group. The relative ratios of PCNA/ $\beta$-actin were significant increased prostates from rats in the group with TP-induced $\mathrm{BPH}$ $(0.62 \pm 0.13, P<0.01)$ compared with those from rats in the NC group $(0.29 \pm 0.21)$. By contrast, rats in the Finand PFE-treated groups $(0.30 \pm 0.13$ and $0.35 \pm 0.16$, $P<0.01$ and $P<0.05$, respectively) exhibited a significantly reduced relative ratio of $\mathrm{PCNA} / \beta$-actin compared with rats in the $\mathrm{BPH}$ group (Fig. 7b).

\section{Effects of PFE on 5a-reductase activity}

To compare the inhibitory activity of PFE, Rib (nonsteroidal positive control) and Fin (steroidal positive control) against enzyme suspension of $5 \alpha$-reductase prepared from prostates of rats, enzyme assay was performed. As shown in Table 1, PFE showed a weak inhibitory activity (31.8 $\pm 15.0 \%)$, whereas nonsteroidal (Rib, $89.1 \pm 18.1 \%$ ) and steroidal (Fin, $94.3 \pm 46.1 \%$ ) positive controls showed potent inhibition.

\section{Discussion}

In the present study, we evaluated the antiproliferative and antioxidant effects of PFE on the development of $\mathrm{BPH}$ using a TP-induced model of BPH in rats. Rats with TP-induced BPH showed an increased relative prostate weight, elevated testosterone and DHT levels, prostatic epithelial hyperplasia, decreased the activities of antioxidant enzymes and overexpression of PCNA. However, we found that oral administration of PFE effectively prevented the progression of BPH caused by testosterone. Treatment of PFE inhibited the development of $\mathrm{BPH}$, which was observed by decreased relative prostate weights, reduced testosterone and DHT levels in serum and prostatic tissue, and inhibited expression of PCNA. Histological changes also indicated that PFE treatment influenced mild prostatic epithelial hyperplasia. In addition, PFE showed mild $5 \alpha$-reductase inhibitory activity.

$\mathrm{BPH}$ is a noncancerous and precancerous prostate condition caused by the overgrowth of prostatic epithelial and stromal cells, which results from an imbalance between proliferation and apoptosis of cells in the prostate [20]. Prostatic enlargement as increased prostate weight has been used as a vital marker of BPH progression [21]. The relative prostate weight used in the present study was the absolute prostate weight to body weight ratio. In this study, $\mathrm{BPH}$ induced by testosterone markedly increased in the prostate volume, which is consistent with previous studies $[8,14]$. Our results demonstrated that PFE treatment significantly decreased the relative prostate weight. Histological examination of prostate tissues paralleled the results of prostate weight measurement. In support of these results, histological findings indicated that administration of PFE 


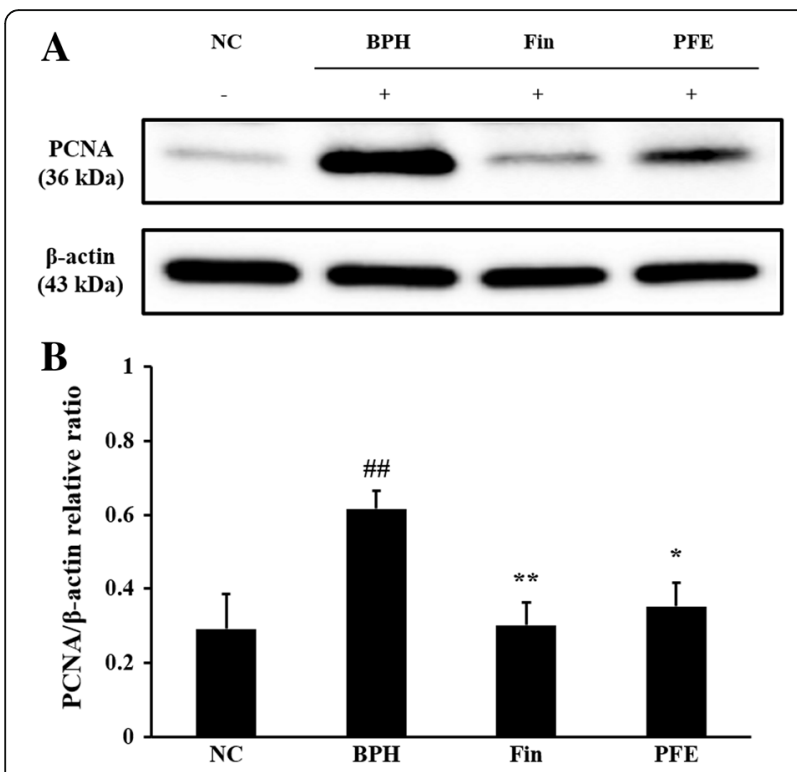

Fig. 7 Effect of PFE on the expression of PCNA in prostatic tissue. Prostatic tissues were homogenized and PCNA/ $\beta$-actin protein expression was determined using western blotting: (a) expressions of PCNA and $\beta$-actin, and (b) relative ratio, relative levels of PCNA. The $\beta$-actin protein was used as a loading control for quantitative analysis. The relative ratios of protein expression were normalized to $\beta$-actin and are represented in quantitative graphs. NC (normal control group), corn oil injection (s.c.) + PBS administration (p.o.); BPH (control group), TP (3 mg/kg)/corn oil injection (s.c.) + PBS administration (p.o.); Fin (positive control group), TP (3 mg/kg)/corn oil injection (s.c.) + finasteride administration (10 mg/kg, p.o.); PFE (PFE-treated group), TP (3 mg/kg)/corn oil injection (s.c.) + PFE administration (200 mg/kg, p.o.). Data are presented as mean \pm S.E.M. $(n=6)$. Significant differences at ${ }^{\# \# P}<0.01$ compared with the NC group. Significant differences at ${ }^{*} P<0.05$ and ${ }^{* *} P<0.01$ compared with the $\mathrm{BPH}$ group. $\mathrm{BPH}$, benign prostatic hyperplasia; Fin, finasteride; PBS, phosphate-buffered saline; PFE, Ponciri fructus extract; p.o., peroral; s.c., subcutaneously; PCNA, proliferating cell nuclear antigen; TP, testosterone propionate

remarkably attenuated the prostatic epithelial hyperplasia, which reduces prostate volume or size.

Testosterone and DHT are steroid hormones related to $\mathrm{BPH}$ and play an important role in the development of internal male reproductive organs $[4,5]$. Testosterone is converted into DHT by the $5 \alpha$-reductase enzyme responsible for the development of the prostate and pathogenesis of BPH. DHT, a derivative of testosterone, stimulates cell

Table 1 Inhibitory effects of PFE, Rib and Fin on 5a-reductase activity in rat prostate

\begin{tabular}{ll}
\hline Group & 5a-reductase inhibition (\%) \\
\hline PFE & $31.8 \pm 15.0$ \\
Rib & $89.1 \pm 18.1$ \\
Fin & $94.3 \pm 46.1$ \\
\hline
\end{tabular}

The effect of PFE (PFE-treated group) on rat prostatic 5a-reductase was tested at a concentration of $0.25 \mathrm{mg} / \mathrm{mL}$. A nonsteroidal-positive control with Rib (riboflavin-treated group) of $1000 \mu \mathrm{M}$ and a steroidal-positive control with Fin (finasteride-treated group) of $100 \mathrm{nM}$. Values are means of results from two different experiments proliferation and growth in the prostate and is a major cause of rapidly enlarged prostate. As such, DHT is considered to be the most important prostatic hormone in development and progression of $\mathrm{BPH}$ [22]. Additionally, steroid hormones and growth factors play a major role in regulating a variety of cellular processes that can lead to cell growth, proliferation, and differentiation [23]. Growth factor-induced PCNA is a major proliferative marker and plays a key role in certain pathological/ physiological processes [7]. Consistent with previous studies [7, 13, 24], we found that administration of PFE significantly decreases the levels of steroid hormones, such as testosterone and DHT, resulting in increased $5 \alpha$-reductase inhibitory activity and attenuating the expression of PCNA. Therefore, these results demonstrate that PFE should be considered as an effective antiproliferation agent in the treatment of prostatic hyperplasia.

Acute or chronic inflammation leads to proliferative events by stimulating cell growth and development through oxidative stress resulting from the overproduction of reactive oxygen species. Antioxidant enzymes, such as CAT, GPx, GR, and SOD normally protect prostatic cells, which activate the body's defense system to damage caused by oxidative stress [25]. Antioxidant enzymes function as scavengers of free radicals by converting them to less harmful oxygen species [26]. Higher activities of antioxidant enzymes have an antioxidant effect by the alleviation of oxidative stress [7, 25]. PFE treatment significantly the increased activities of antioxidant enzymes responsible for the reduction of oxidative damage in prostatic tissue induced by testosterone. Thus, PFE could effectively reverse the changes in activities of antioxidant enzymes, which act as antioxidant agents.

Ponciri Fructus is a widely used a traditional herbal medicine. It has been reported that three flavonoids (naringin, neoponcirin and poncirin) and three coumarins (umbelliferone, imperatorin and auraptene) isolated from the $70 \%$ ethanolic extract of Ponciri fructus have various pharmacological actions including antioxidant [27], anxiolytic-like [28], anticancer [29], and anti-inflammatory effects [30-32]. Therefore, we propose that PFE can have antiproliferative and antioxidant effects based on the various pharmacological activities of the chemical-chemical interactions on the development and progression of lesions in a TP-induced model of BPH.

\section{Conclusions}

In summary, the findings of our present study demonstrated that PFE significantly reduced the relative prostate weight, prostatic hyperplasia, level of testosterone and DHT closely related to $5 \alpha$-reductase inhibitory activity, expression of PCNA, and significantly increased activities of antioxidant enzymes. These findings suggest that PFE exerts antiproliferative and antioxidant effects in preventing the development and progression of $\mathrm{BPH}$. 


\section{Abbreviations}

ANOVA: analysis of variance; BPH: Benign prostatic hyperplasia; CAT: catalase; DHT: dihydrotestosterone; ELISA: enzyme-linked immunosorbent assay; Fin: Finasteride; GPx: glutathione peroxidase; GR: glutathione reductase; HPLC: High-performance liquid chromatography; p.o:: peroral; PBS: phosphate buffered saline; PCNA: proliferating cell nuclear antigen; PFE: Ponciri Fructus extract (PFE); Rib: riboflavin; s.c.: subcutaneously; SD: Sprague Dawley; SEM: standard error of the mean; SOD: superoxide dismutase; TP: testosterone propionate

\section{Acknowledgments}

This study was supported by the Convergence of Conventional Medicine and Traditional Korean Medicine R\&D program (H16C0778) funded by the Ministry of Health \& Welfare through the Korea Health Industry Development Institute (KHIDI).

\section{Funding}

Convergence of Conventional Medicine and Traditional Korean Medicine R\&D program (H16C0778) funded by the Ministry of Health \& Welfare through the Korea Health Industry Development Institute (KHIDI).

\section{Availability of data and materials}

All data supporting our findings are adequately contained within the manuscript.

\section{Authors' contributions}

Woo-Young Jeon, Hyeun-Kyoo Shin, Yong-ung Kim, and Mee-Young Lee participated in the design of the data analyses and manuscript preparation. Woo-Young Jeon, Ohn Soon Kim, Chang-Seob Seo, Seong Eun Jin and Jung-Ae Kim conducted the assays and analyses. All authors read and approved the final manuscript.

\section{Ethics approval}

The study was approved by the Institutional Animal Care and Use Committee of the Chungnam National University (animal ethics approval number: CNU-00446).

\section{Consent for publication}

Not applicable.

\section{Competing interests}

The authors declare that there is no conflict of, or competing financial interests regarding, the publication of this paper.

\section{Publisher's Note}

Springer Nature remains neutral with regard to jurisdictional claims in published maps and institutional affiliations.

\section{Author details}

${ }^{1} \mathrm{~K}$-herb Research Center, Korea Institute of Oriental Medicine, 1672 Yuseong-daero, Yuseong-gu, Daejeon 34054, Republic of Korea. ${ }^{2} \mathrm{KM}$ Convergence Research Division, Korea Institute of Oriental Medicine, 1672 Yuseong-daero, Yuseong-gu, Daejeon 34054, Republic of Korea. ${ }^{3}$ School of Pharmacy, College of Pharmacy, Yeungnam University, 280 Daehak-ro, Gyeongsan-si, Gyeongsangbuk-do 38541, Republic of Korea. ${ }^{4}$ Department of Pharmaceutical Engineering, College of Biomedical Science, Daegu Haany University, 1 Hanuidae-ro, Gyeongsan-si, Gyeongsangbuk-do 38610, Republic of Korea.

Received: 9 April 2017 Accepted: 9 July 2017

Published online: 03 August 2017

\section{References}

1. Untergasser G, Madersbacher S, Berger P. Benign prostatic hyperplasia: age-related tissue-remodeling. Exp Gerontol. 2005;40:121-8. doi:10.1016/ j.exger.2004.12.008.

2. Roehrborn CG. Male lower urinary tract symptoms (LUTS) and benign prostatic hyperplasia (BPH). Med Clin North Am. 2011;95:87-100. doi:10.1016/j.mena.2010.08.013.

3. Lucia MS, Lambert JR. Growth factors in benign prostatic hyperplasia: basic science implications. Curr Urol Rep. 2008;9:272-8.

4. Andriole G, Bruchovsky N, Chung LW, Matsumoto AM, Rittmaster R, Roehrborn C, Russell D, Tindall D. Dihydrotestosterone and the prostate: the scientific rationale for 5alpha-reductase inhibitors in the treatment of benign prostatic hyperplasia. J Urol. 2004;172:1399-403.

5. Carson C 3rd, Rittmaster R. The role of dihydrotestosterone in benign prostatic hyperplasia. Urology. 2003;61:2-7.

6. Bostanci Y, Kazzazi A, Momtahen S, Laze J, Djavan B. Correlation between benign prostatic hyperplasia and inflammation. Curr Opin Urol. 2013;23:5-10. doi:10.1097/MOU.0b013e32835abd4a.

7. Sarbishegi M, Khani M, Salimi S, Valizadeh M, Sargolzaei Aval F. Antiproliferative and antioxidant effects of Withania Coagulans extract on benign prostatic hyperplasia in rats. Nephrourol Mon. 2016:8:e33180. doi:10.5812/numonthly.33180.

8. Yang X, Yuan L, Xiong C, Yin C, Ruan J. Abacopteris penangiana exerts testosterone-induced benign prostatic hyperplasia protective effect through regulating inflammatory responses, reducing oxidative stress and antiproliferative. J Ethnopharmacol. 2014;157:105-13. doi:10.1016/j.jep.2014.09.025.

9. KAPE: Handbook of the Korea Pharmacopoeia. 9th ed. Shinilbooks. Korea: Seoul; 2008. p. 1176-7

10. Shin EM, Zhou HY, Xu GH, Lee SH, Merfort I, Kim YS. Anti-inflammatory activity of hispidol a 25-methyl ether, a triterpenoid isolated from Ponciri Immaturus Fructus. Eur J Pharmacol. 2010;627:318-24. doi:10.1016/j.ejphar.2009.10.036.

11. Lee JH, Lee SH, Kim YS, Jeong CS. Protective effects of neohesperidin and poncirin isolated from the fruits of Poncirus trifoliata on potential gastric disease. Phytother Res. 2009;23:1748-53. doi:10.1002/ptr.2840.

12. Seo CS, Jeong SJ, Yoo SR, Lee NR, Shin HK: Quantitative Analysis and In vitro Anti-inflammatory Effects of Gallic Acid, Ellagic Acid, and Quercetin from Radix Sanguisorbae. Pharmacogn Mag 2016, 12:104-108. doi:10.4103/ 0973-1296.177908

13. Shin IS, Lee MY, Jung DY, Seo CS, Ha HK, Shin HK. Ursolic acid reduces prostate size and dihydrotestosterone level in a rat model of benign prostatic hyperplasia. Food Chem Toxicol. 2012;50:884-8. doi:10.1016/j.fct.2012.01.007.

14. Veeresh Babu SV, Veeresh B, Patil AA, Warke YB. Lauric acid and myristic acid prevent testosterone induced prostatic hyperplasia in rats. Eur J Pharmacol. 2010;626:262-5. doi:10.1016/j.ejphar.2009.09.037.

15. Jeon WY, Shin IS, Shin HK, Lee MY. Gastroprotective effect of the traditional herbal medicine, Sipjeondaebo-tang water extract, against ethanol-induced gastric mucosal injury. BMC Complement Altern Med. 2014;14:373. doi:10.1186/1472-6882-14-373.

16. Lim HS, Yeji K, Seo CS, Yoo SR, Jin SE, Shin HK, Jeong SJ: Chungsimyeonjaeum inhibits inflammatory responses in RAW 264.7 macrophages and HaCaT keratinocytes. BMC Complement Altern Med 2015, 15:371. doi: 10.1186/s12906-015-0902-2.

17. Kim S, Kim Y, Ma E. Synthesis and 5a-reductase inhibitory activity of C21 steroids having 1,4-diene or 4,6-diene 20-ones and 4-azasteroid 20-oximes. Molecules. 2011;17:355-68. doi:10.3390/molecules17010355.

18. Russell DW, Wilson JD. Steroid 5 alpha-reductase: two genes/two enzymes. Annu Rev Biochem. 1994;63:25-61. doi:10.1146/annurev.bi.63.070194.000325.

19. Dunnett CW. New tables for multiple comparisons with control. Biometrics. 1964;20:482-91.

20. Afriyie DK, Asare GA, Bugyei K, Adjei S, Lin JM, Peng J, Hong ZF. Treatment of benign prostatic hyperplasia with Croton Membranaceus in an experimental animal model. J Ethnopharmacol. 2014;157:90-8. doi:10.1016/j.jep.2014.09.007.

21. Pais P. Potency of a novel saw palmetto ethanol extract, SPET-085, for inhibition of 5alpha-reductase II. Adv Ther. 2010;27:555-63. doi:10.1007/s12325-010-0041-6.

22. Nicholson TM, Ricke WA. Androgens and estrogens in benign prostatic hyperplasia: past, present and future. Differentiation. 2011:82:184-99. doi:10.1016/j.diff.2011.04.006

23. Ciarmela P, Islam MS, Reis FM, Gray PC, Bloise E, Petraglia F, Vale W, Castellucci M. Growth factors and myometrium: biological effects in uterine fibroid and possible clinical implications. Hum Reprod Update. 2011;17:772-90. doi:10.1093/humupd/dmr031.

24. Shen HN, Xu Y, Jiang ZZ, Huang X, Zhang LY, Wang T. Inhibitory effects of Tripterygium wilfordii multiglycoside on benign prostatic hyperplasia in rats. Chin J Nat Med. 2015;13:421-7. doi:10.1016/S1875-5364(15)30035-2.

25. Ammar AE, Esmat A, Hassona MD, Tadros MG, Abdel-Naim AB, Guns ES. The effect of pomegranate fruit extract on testosterone-induced $\mathrm{BPH}$ in rats. Prostate. 2015;75:679-92. doi:10.1002/pros.22951.

26. Sies H. Strategies of antioxidant defense. Eur J Biochem. 1993;215:213-9.

27. Kumar VS, Rajmane AR, Adil M, Kandhare AD, Ghosh P, Bodhankar SL. Naringin ameliorates acetic acid induced colitis through modulation of endogenous oxido-nitrosative balance and DNA damage in rats. J Biomed Res. 2014;28:132-45. doi:10.7555/JBR.27.20120082. 
28. Cassani J, Araujo AG, Martínez-Vázquez M, Manjarrez N, Moreno J, EstradaReyes R. Anxiolytic-like and antinociceptive effects of 2(S)-neoponcirin in mice. Molecules. 2013;18:7584-99. doi:10.3390/molecules18077584.

29. Saralamma W, Nagappan A, Hong GE, Lee HJ, Yumnam S, Raha S, Heo JD, Lee SJ, Lee WS, Kim EH, Kim GS. Poncirin induces apoptosis in AGS human gastric cancer cells through extrinsic apoptotic pathway by up-regulation of Fas ligand. Int J Mol Sci. 2015;16:22676-91. doi:10.3390/ijms160922676.

30. Sim MO, Lee HI, Ham JR, Seo KI, Kim MJ, Lee MK. Anti-inflammatory and antioxidant effects of umbelliferone in chronic alcohol-fed rats. Nutr Res Pract. 2015;9:364-9. doi:10.4162/nrp.2015.9.4.364.

31. Guo W, Sun J, Jiang L, Duan L, Huo M, Chen N, Zhong W, Wassy L, Yang Z, Feng $\mathrm{H}$. Imperatorin attenuates LPS-induced inflammation by suppressing NF-KB and MAPKs activation in RAW 264.7 macrophages. Inflammation. 2012;35:1764-72. doi:10.1007/s10753-012-9495-9.

32. Okuyama S, Morita M, Kaji M, Amakura Y, Yoshimura M, Shimamoto K, Ookido Y, Nakajima M, Furukawa Y. Auraptene acts as an anti-inflammatory agent in the mouse brain. Molecules. 2015;20:20230-9. doi:10.3390/ molecules201119691.

Submit your next manuscript to BioMed Central and we will help you at every step:

- We accept pre-submission inquiries

- Our selector tool helps you to find the most relevant journal

- We provide round the clock customer support

- Convenient online submission

- Thorough peer review

- Inclusion in PubMed and all major indexing services

- Maximum visibility for your research

Submit your manuscript at www.biomedcentral.com/submit
Biomed Central 